\title{
Low-dose Prasugrel in Patients with Resistance to Clopido- grel for the Treatment of Cerebral Aneurysms: Follow-up of over 6 Months
}

\author{
Dongwhane Lee, MD, Yunsun Song, MD, Jae Ho Shin, MD, Dae Chul Suh, MD, PhD \\ Neurointervention Clinic, Department of Radiology, Asan Medical Center, University of Ulsan College of Medicine, Seoul, Korea
}

We recently reported the use of a lowdose of prasugrel for coil embolization of unruptured cerebral aneurysms. Twenty-four patients showing resistance to clopidogrel using a $\mathrm{P} 2 \mathrm{Y} 12$ reaction unit (PRU) test, were treated with a 20 mg loading dose of prasugrel followed by a 5 mg maintenance dose. No thromboembolic event or hemorrhagic complication was noted during the mean follow-up period of 1.5 months. Herein, we report the results of our observational cases during a more than 6-month follow-up regarding the use of prasugrel for a short-term period of administration.

The median follow-up period was 221 days (interquartile range [IQR], 202.25294.00) in the 24 patients. Patients treated with stent-assisted coil embolization $(\mathrm{n}=18)$ received aspirin for 220 days (IQR, 200-267.5) and prasugrel for 90 days (IQR, 60-125). Patients who underwent only coil embolization $(n=6)$ were administered aspirin for only for 40 days (IQR, 10-40). This was a modified protocol involving the lifelong use of aspirin after 3 months of short-term administration of prasugrel in patients who underwent stent-assisted procedures. ${ }^{2}$

One patient (female, 67 years old) who underwent stent-assisted coil embolization for aneurysm of an anterior communicating (A-com) artery was enrolled in our study despite prasugrel not being used because of the intension-to-treat study design. The aspirin reactive unit (ARU) at admission was 398, and the PRU value was 218 with a percentage inhibition of $0 \%$. After a loading dose of prasugrel, the PRU and percentage inhibition values were 154 and 39\%, respectively, which indicated that the patient was responding. An evaluation of her medical history revealed pontine infarction two months ago. She was on medication for one year due to hypertension and hyperlipidemia. In addition, echocardiography and transthoracic echocardiogram revealed normal findings. Since the uses of prasugrel is not recommended in patients with low (50-kg) body weight and with history of ischemic stroke, we changed the prescription to aspirin and cilostazol for this patient. ${ }^{3}$ After successful stent-assisted coil embolization of a 9-mm A-com artery aneurysm, she developed an acute ischemic stroke 9 days after the procedure (Fig. 1). She visited the emergency room with a complaint of left hemiparesis, and an acute infarction of the right

\author{
Correspondence to: \\ Dae Chul Suh, MD, PhD \\ Neurointervention Clinic, Department \\ of Radiology, Asan Medical Center, \\ University of Ulsan College of Medi- \\ cine, 88 Olympic-ro 43-gil, Songpa-gu, \\ Seoul 05505, Korea \\ Tel: +82-2-3010-4366 \\ Fax: +82-2-3010-0090 \\ E-mail:dcsuh@amc.seoul.kr \\ Received: February 15, 2019 \\ Revised: February 22, 2019 \\ Accepted: February 22, 2019
}

\begin{abstract}
Copyright $\odot 2019$ Korean Society of Interventional Neuroradiology This is an Open Access article distributed under the terms of the Creative Commons Attribution Non-Commercial License (http://creativecommons.org/licenses/by-nc/3.0) which permits unrestricted non-commercial use, distribution, and reproduction in any medium, provided the original work is properly cited.
\end{abstract}

pISSN 2093-9043 eISSN 2233-6273 

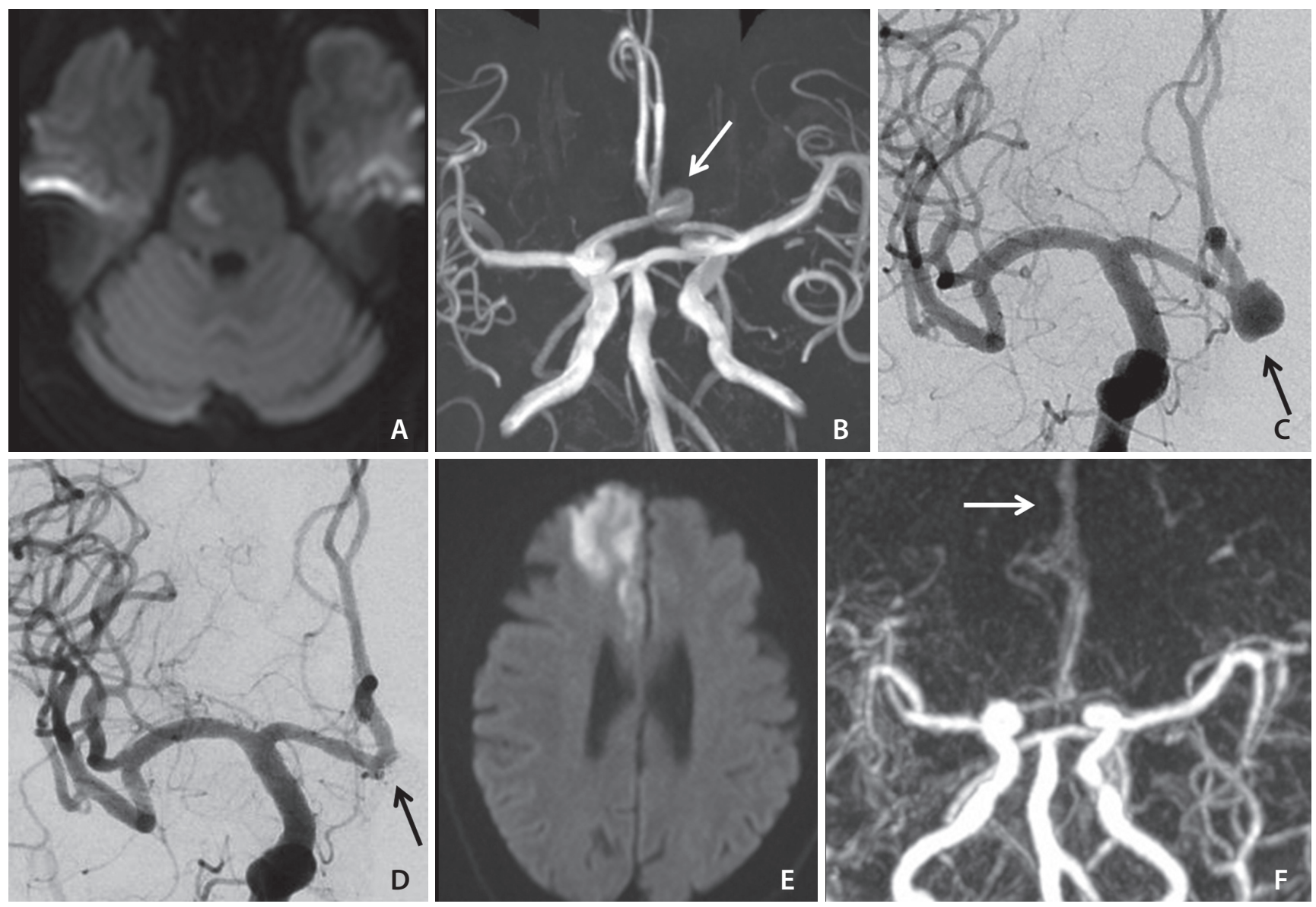

Fig. 1. A thromboembolic event in a patient with 50-kg body weight under aspirin and cilostazol due to clopidogrel resistance. Prasugrel was not prescribed due to low body weight and a history of pontine infarction 2 months ago (A). (B) Incidental aneurysm is found in the anterior communicating artery (white arrow). (C) Pre- and (D) post-embolization cerebral angiograms. Stent-assisted coil embolization was performed for the aneurysm (black arrow). (E) Diffusion-weighted image shows an acute infarction in the anterior cerebral artery territory. (F) Magnetic resonance angiography shows a faint filling of the anterior cerebral artery. Revascularization was not tried. Final modified Rankin Scale score was 2 at 90 days.

anterior cerebral artery (ACA) territory was observed in the magnetic resonance-diffusion weighted image. Magnetic resonance angiography revealed faint filling of the ACA; we did not attempt recanalization therapy because dense infarction was already noted in the right ACA territory on apparent diffusion coefficient map and fluid-attenuated inversion recovery image. We regarded the cause of cerebral infarction as atherothrombotic rather than cardioembolic because previous cardiac studies were normal. Modified Rankin Scale 90 days was 2. Other minor complications associated and observed with the use of prasugrel included easy bruising in one patient and menorrhagia in another.

Prasugrel is a fast, potent, relatively safe and effective alternative to be used in patients with resistance to clopidogrel for the premedication for unruptured intracranial aneurysms. ${ }^{2,4}$ However, in patients with a recent history of ischemic stroke, the choice of antiplatelet agents is challenging because these patients have not been responding to their therapy and prasugrel is contraindicated in these patients, due to the increased risk of potential hemorrhage. Further discussion is needed to determine the reason for the limited use of prasugrel in patients aged 75 years or older and in those weighing less than $60 \mathrm{~kg}^{3}$ In our practice, we recommend omitting one or two doses per week in patients presenting with a hyper-response to prasugrel and with low body weight, in order to prevent any hemorrhagic complications. Proper monitoring of the antiplatelet response for patient weighting less than $60 \mathrm{~kg}$ and in needs to receive prasugrel and may allow to prescribe the proper dosage without increasing the risk of complications, in this population of patients. Moreover, the pharmacological mechanism needs to be clarified to understand why further reduction of 
dose is not possible in patients weighing less than $60 \mathrm{~kg}$.

In conclusion, the use of low dose of prasugrel has been providing us with an alternative when patients present with a low or non-response to clopidogrel. More data still will be needed to optimize antiplatelets regimen in our population of patients.

\section{Acknowledgments}

This work was supported by the National Research Foundation of Korea (NRF) grant funded by the Korea government (MSIT; No. 2018R1A2B6003143).

\section{REFERENCES}

1. Lee $D$, Song $Y$, Han M, Park D, Suh DC. Low-dose prasugrel in patients with resistance to clopidogrel for the treatment of cerebral aneurysms. Neurointervention 2018;13:124-127

2. Choi HH, Lee JJ, Cho YD, Han MH, Cho WS, Kim JE, et al. Antiplatelet premedication for stent-assisted coil embolization of intracranial aneurysms: low-dose prasugrel vs clopidogrel. Neurosurgery 2018;83:981-988

3. Wiviott SD, Braunwald E, McCabe CH, Montalescot G, Ruzyllo W, Gottlieb S, et al. Prasugrel versus clopidogrel in patients with acute coronary syndromes. N Engl J Med 2007;357:2001-2015

4. Sedat J, Chau Y, Gaudart J, Sachet M, Beuil S, Lonjon M. Prasugrel versus clopidogrel in stent-assisted coil embolization of unruptured intracranial aneurysms. Interv Neuroradiol 2017;23:52-59 